Classification

Physics Abstracts

08.45

\title{
DISPOSITIFS ORIGINAUX D'UN MONOCHROMATEUR INFRAROUGE (1-33 $\mu$ ) POUR ÉTUDES MULTIPLES
}

\author{
M. CADENE et C. BENOIT (*) \\ Laboratoire de Physique Moléculaire et Cristalline \\ Faculté des Sciences, 34, Montpellier \\ (Reçu le 2 mars 1972, révisé le 18 juillet 1972)
}

\begin{abstract}
Résumé. - Nous avons construit un monochromateur infrarouge à performances élevées permettant d'explorer une zone du spectre s'étendant de $1 \mu$ à $33 \mu$. Cet appareil, de conception classique, comporte des dispositifs optiques et mécaniques originaux. Il a été utilisé avec succès dans le cas des études des propriétés anharmoniques des cristaux ioniques et moléculaires ainsi que pour préciser les structures de certains cristaux.

Abstract. - We have built a high performance infrared monochromator allowing to measure the $1 \mu-33 \mu$ range spectra. This classical instrument presents some new optical and mechanical devices. We have used it to study the anharmonicity of ionic and molecular compounds, and the structures of some crystals.
\end{abstract}

Dans le domaine des études portant sur l'état solide (structures, dynamique du réseau cristallin), il s'avère indispensable d'effectuer des mesures de plus en plus précises et de type différent, telles que transmission, réflexion en lumière polarisée de monocristaux soumis à des contraintes multiples (température, pression, excitations extérieures intenses).

A ce titre, dès 1956, plusieurs appareils ont été construits [1] au laboratoire ou modifiés [2] sur le principe original des blocs adaptables permettant d'effectuer tous les types de mesures désirés, par simple changement de la platine de mesure ou d'un de ses organes, ce principe ayant été en définitive adopté par l'industrie et commercialisé depuis quelques années. Bénéficiant de l'expérience acquise, nous avons conçu et réalisé un nouvel appareil, dont nous décrivons ici quelques dispositifs originaux.

Ses caractéristiques générales sont les suivantes:

- Monochromateur à réseau et prisme d'ouverture $F / 7$ utilisable de $1 \mu$ à $33 \mu\left(10000-300 \mathrm{~cm}^{-1}\right)$. Défilement linéaire en longueur d'onde. Polariseur à lames de sélénium.

- Utilisation en simple ou multiple faisceau, en défilement continu ou point par point.

- Interchangeabilité rapide et précise des sources, des éléments dispersifs et des récepteurs.

- Platine de mesures (réflexion, transmission, à température variable et sous pression...).

- Eclairement de l'échantillon en lumière monochromatique.

(*) Adresse actuelle : Département de Physique, Faculté des Sciences, 64016, Pau.
L'ensemble du spectromètre est placé dans une enceinte permettant un balayage d'air sec.

$\mathrm{Au}$ cours de la description rapide du schéma optique et des mécanismes de commandes et de programmation des mesures, nous insisterons plus particulièrement sur les dispositifs qui, outre le principe des blocs interchangeables que nous avons toujours préconisé, font l'originalité de ce monochromateur.

Les performances de l'appareil seront ensuite discutées et illustrées par quelques travaux originaux menés sur différents corps.

\section{A. DISPOSITION OPTIQUE}

Etabli dans le souci constant de ne présenter que le minimum d'aberrations, l'ensemble comprend trois blocs interchangeables, mais rigidement liés (Fig. 1) :

- Bloc monochromateur.

- Bloc récepteur.

- Platine de mesures.

I. Monochromateur. - 1) SOURCES ET MONOCHROMATEUR A RÉSEAU : Lampes Philips à filament plan et globar sont placés côte à côte dans une enceinte refroidie constituant le bloc sources (passage de l'une à l'autre par translation). Le faisceau émis est modulé à une fréquence de 11 cycles par seconde avant d'être concentré sur la fente d'entrée du monochromateur proprement dit. Ce système de modulation «en tête » évite les perturbations provenant de l'émission des pales du modulateur.

Pour la meilleure utilisation des réseaux «échelettes» par réflexion, de surface utile égale à $102 \times 128 \mathrm{~mm}$, 


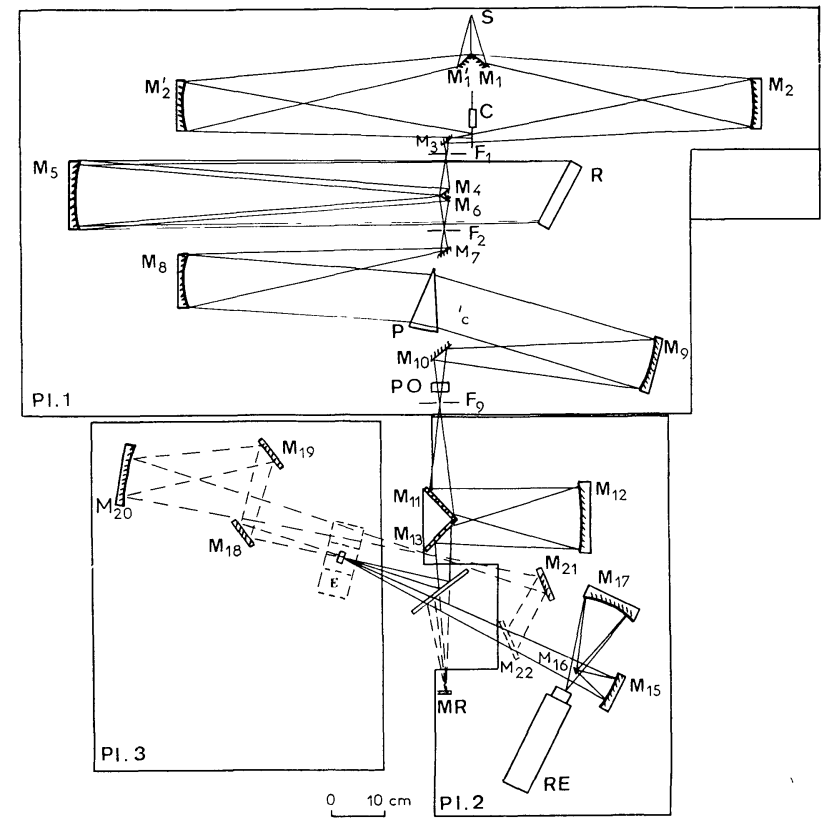

FIG. 1. - Schéma du dispositif optique du spectromètre.

notre choix, guidé par les travaux antérieurs [1]-[7] s'est fixé sur un montage de type LITTROW avec un dispositif d'entraînement linéaire en longueur d'onde.

Ce montage autocollimateur utilisant le même miroir pour les faisceaux incident et diffracté fait travailler le réseau de part et d'autre de l'angle de miroitement. Il reste de plus très lumineux surtout si on limite légèrement le domaine utilisable dans chaque ordre du réseau (variation de $10^{\circ} \mathrm{de}$ part et d'autre de cet angle [5]).

Il est ici essentiellement particularisé par une absence quasi totale d'aberrations due à une symétrie parfaite des éléments, et à une inclinaison très faible, de l'ordre d'un degré, des faisceaux sur l'axe optique du miroir collimateur $\mathbf{M}_{5}$.

a) Miroirs centraux. - Les deux miroirs plans centraux $M_{4}$ et $M_{6}(20 \mathrm{~mm} \times 20 \mathrm{~mm}$, biseautés à $45^{\circ}$ ) sont placés très près de l'axe réseau-miroir $M_{5}$. Cette position originale permet à l'ensemble du dispositif optique d'être situé dans le même plan horizontal (Fig. 2), de travailler très près des conditions de Gauss (minimum d'aberrations) et d'utiliser des fentes rectilignes de grande hauteur (jusqu'à $15 \mathrm{~mm}$ ), facilement usinées avec précision. L'image de la fente d'entrée donnée par le système est de très bonne qualité et nous atteignons, pour une largeur donnée, le pouvoir de résolution utile de l'appareil.

L'ombre portée par les miroirs $\mathbf{M}_{4}$ et $\mathbf{M}_{6}$ sur le réseau produit une diminution de flux de l'ordre de $6 \%$ mais cet inconvénient est largement compensé par la résolution obtenue et la simplicité de construction et de mise au point du système.

La fraction du flux incident non diffractée, réfléchie directement par le miroir $\mathbf{M}_{6}$, forme une image de la fente d'entrée au foyer du miroir sphérique $M_{8}$.

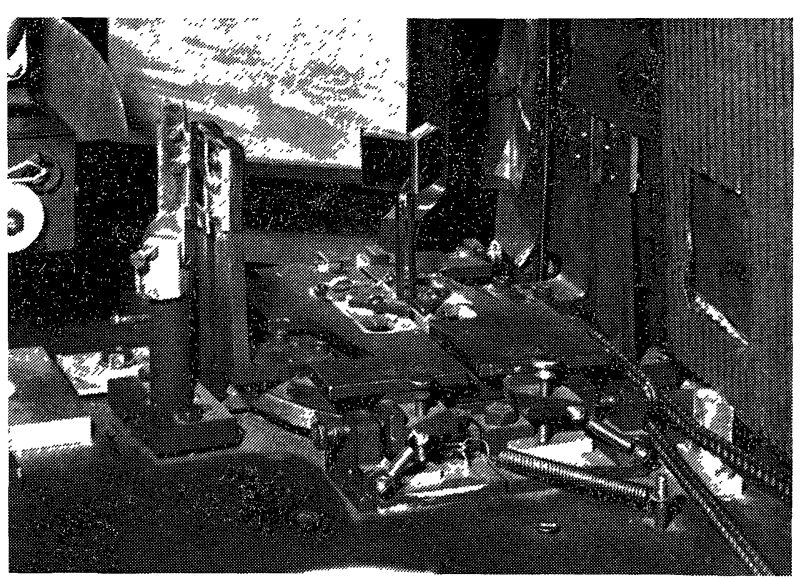

FIg. 2. - Miroirs centraux. Ouverture symétriqua des fentes.

Cette image parasite est éliminée très facilement par un cache, qui n'entraîne d'ailleurs pas de perte de flux supplémentaire, celui-ci étant placé dans l'ombre portée des miroirs $\mathbf{M}_{4}$ et $\mathbf{M}_{6}$.

b) Support de réseaux et disposition des fentes. Nous avons été conduits :

- à imaginer un support de réseaux totalement original (Fig. 3), permettant l'interchangeabilité rapide

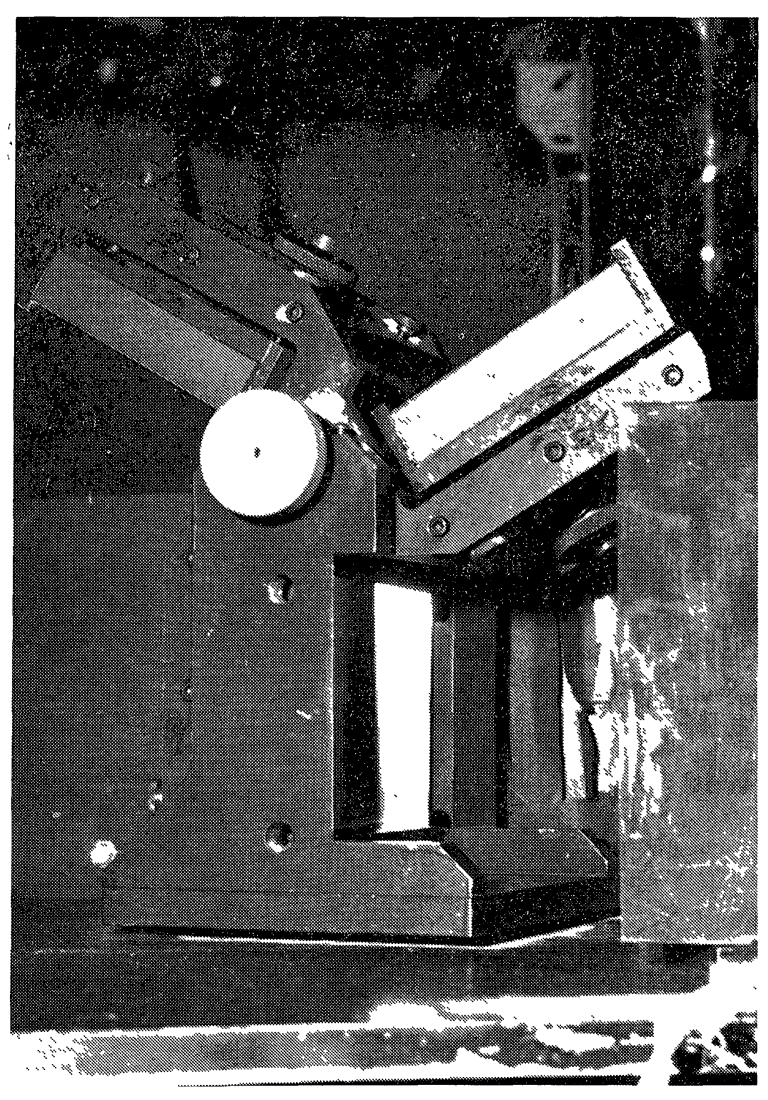

FIG. 3. - Support des réseaux.

et précise de trois réseaux, par simple rotation autour d'un axe horizontal. Les deux réseaux non utilisés sont en effet placés au-dessus du $3^{\mathrm{e}}$ réseau, leurs sur- 
faces étant à $120^{\circ}$ l'une de l'autre. Le réseau en fonction est bloqué par clavette et butée:

Ce dispositif a l'avantage, outre la rapidité exceptionnelle du changement, de placer l'axe vertical de rotation du réseau dans le plan de sa surface et d'assurer une reproductibilité parfaite de l'étalonnage ;

- à utiliser une disposition particulière des fentes d'entrée et de sortie (Fig. 2) dont l'ouverture simultanée et symétrique est obtenue par la rotation en sens inverse de deux plates-formes horizontales circulaires montées sur des axes coaxiaux. Ces plates-formes portent chacune, symétriquement par rapport au centre, une des lèvres des fentes $F_{1}$ et $F_{2}$ et sont reliées par une chaînette tendue sur une poulie à excentrique. La rotation de l'axe interne solidaire de la plate-forme supérieure détermine l'ouverture des fentes. Cette rotation peut être soit manuelle, soit synchronisée à la rotation du réseau. Nous nous sommes par la suite aperçus que ce dispositif d'ouverture symétrique était utilisé sur un spectromètre déjà commercialisé.

2) SÉPARATEUR D'ORDRE. — La séparation des ordres est obtenue à l'aide de prismes de petit angle (trois prismes interchangeables $\mathrm{NaCl}, \mathrm{KBr}, \mathrm{CsBr}$, angle d'environ $30^{\circ}$ ). Le miroir $\mathrm{M}_{9}$ est entraîné en rotation (Fig. 4), en liaison avec le mouvement du réseau. L'inclinaison du rayon moyen sur la normale au miroir $\mathrm{M}_{8}$ ou $\mathrm{M}_{9}$ est de l'ordre de $6^{\circ}$.
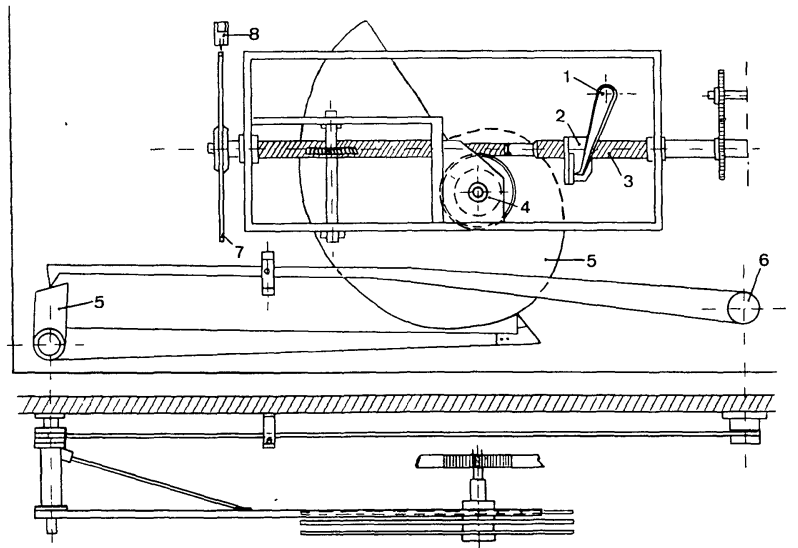

Fig. 4. - Ensemble des mécanismes d'entraînement. 1. Axe réseau ; 2. Chariot ; 3 . Vis ; 4. Axe de synchronisation; 5. Cames du séparateur d'ordres; 6 . Axe miroir séparateur d'ordres;

7. Roue dentée ; 8. Electro-aimant de l'échappement à ancre.

Entre le miroir $\mathrm{M}_{10}$ et la fente $\mathrm{F}_{3}$, un polariseur à lames minces de sélénium peut venir s'intercaler sur le faisceau. Cette position du polariseur à la sortie $\mathrm{du}$ bloc spectromètre, juste avant l'échantillon, permet d'éviter les différents effets non commutatifs de polarisation dus aux éléments dispersifs et aux miroirs.
II. Bloc récepteur. - Les montages très diversifiés de l'échantillon sur la platine de mesures sont possibles grâce à certains éléments importants de la platine portant le bloc récepteur.

a) Système à convergence variable. - Le faisceau issu de la fente de sortie du spectromètre tombe sur un ensemble de deux miroirs plans $M_{11}$ et $M_{13}$ et d'un miroir sphérique $\mathbf{M}_{12}$, permettant de faire varier sa convergence entre $5^{\circ}$ et $15^{\circ}$ par le déplacement précis en translation, soit de l'ensemble, soit du miroir sphérique seul.

Ce dispositif est particulièrement utile, outre le cas de l'étude de cristaux de faibles dimensions, pour les mesures où un appareillage important entoure l'échantillon (mesures à basse température, effets de pression). La suppression du miroir $\mathrm{M}_{13}$ permet de plus une sortie directe du faisceau, nécessaire vu l'encombrement de la presse, pour les mesures aux hautes pressions.

b) Disque tournant. - Suivant le système à convergence variable, un disque tournant comportant deux miroirs plans, dont un réglable, et deux ouvertures, permet de diriger le faisceau vers la platine de mesures, donc vers l'échantillon ou, après rotation de $180^{\circ}$, vers un miroir de référence. Les deux positions, échantillon et miroir de référence, sont symétriques par rapport au disque tournant et situées au point de convergence du faisceau. Le miroir de référence est réglable en translation (cas d'un changement de convergence du faisceau).

La synchronisation du mouvement de ce disque avec un défilement discontinu du spectre permet la comparaison immédiate des 2 faisceaux 'pour chaque longueur d'onde.

c) Système récepteur. - Plusieurs systèmes récepteurs sont prévus. Pour un récepteur type GOLAY UNICAM ou ONERA, le système de concentration très particulier comporte deux miroirs sphériques concaves $M_{15}$ et $M_{17}$ de courte focale et un miroir plan à $45^{\circ}$ de très faibles dimensions $\mathbf{M}_{16}$. Le grandissement de l'ensemble est égal à $\frac{1}{6}$.

III. Platine de mesure. - La mise en place d'un cryostat ou d'une presse autour du cristal représente un encombrement très important, c'est pourquoi le faisceau monochromatique polarisé après changement éventuel de sả convergence est dévié par le disque tournant et converge en un point $\mathrm{E}$ extérieur aux deux platines monochromateur et «bloc récepteur », où sera situé l'échantillon.

En ce point nous pouvons disposer un secteur porte-cristaux (1) ou une platine de mesures permettant en particulier par une adaptation très rapide du montage optique :

a) La détermination des spectres de réflexion des cristaux en incidence proche de la normale $\left(6^{\circ}\right)$. Un 
porte-échantillon placé en $\mathrm{E}$ est alors associé au système disque tournant-miroir de référence.

b) Des mesures en simple transmission. Le faisceau est renvoyé sur le récepteur sans changement de convergence en suivant le trajet $\mathrm{E}-\mathrm{M}_{18}-\mathrm{M}_{19}-\mathrm{M}_{20^{-}}$ $\mathrm{M}_{21}-\mathrm{M}_{22}$.

c) Des mesures en réflexion avec un angle d'incidence de $6^{\circ}$ ou en transmission, à température variable $\left(295^{\circ} \mathrm{K}-3^{\circ} \mathrm{K}\right)$.

Au-dessus de la platine est suspendu un cryostat à fenêtres, réglable en toutes directions. Afin de comparer dans les mêmes conditions le faisceau échantillon et le faisceau de référence, le cristal est fixé sur une moitié de la fenêtre du porte-échantillon (queue du cryostat), l'autre moitié étant occupée par un miroir (mesures en réflexion) ou inoccupée (mesures en transmission). La comparaison des deux faisceaux s'effectue par une translation prédéterminée du cryostat dans son ensemble (commande par came).

Ce montage présente l'avantage important de permettre le réglage du cristal après la mise en froid, tenant compte ainsi des déformations de la queue porte-échantillon.

d) Des mesures sous pressions. Le faisceau suit alors le trajet direct $\mathrm{M}_{12}$-échantillon- $\mathrm{M}_{18}-\mathrm{M}_{19^{-}} \mathrm{M}_{20^{-}}$ $\mathrm{M}_{21}-\mathrm{M}_{22}$.

\section{B. MÉCANISMES}

Le mécanisme (Fig. 4), fixé sous la platine, est constitué d'une vis creuse, parallèle à l'axe du miroir collimateur, entraînant le déplacement d'un chariot lié à la rotation du réseau, et permettant, outre un défilement linéaire en longueur d'onde, la synchronisation de ce mouvement, après multiplication convenable, avec celui du miroir $\mathrm{M}_{9}$ du séparateur d'ordre, et des cames de programmation d'ouverture des fentes. Une extrémité de la vis porte une roue dentée de grand diamètre permettant un arrêt précis de la rotation pour l'entraînement discontinu. L'autre extrémité est liée à la platine des moteurs, comportant le bloc d'entraînement du spectromètre à différentes vitesses (moteurs synchrones) un embrayage électromagnétique jouant un rôle important dans les mesures point par point, et les dispositifs de repérage des longueurs d'onde. Un programmateur à galettes entraîné par moteur synchrone assure :

a) La commande périodique d'un échappement à ancre, monté face à la route dentée $(\varphi=290 \mathrm{~mm}$, 200 dents) placée en bout de la vis d'entraînement. L'ensemble, en liaison avec l'embrayage électromagnétique de la platine des moteurs, permet l'arrêt précis du spectromètre, à intervalles de longueur d'onde égaux et prédéterminés, sans aucune dérive au cours d'un enregistrement complet point par point. L'avancement dent par dent permet, par exemple avec le 1 er ordre du réseau I (miroitement à $3 \mu$ ), un déplacement $\Delta \lambda=3 \times 10^{-+} \mu$, soit $\Delta v=0,3 \mathrm{~cm}^{-1}$ à $3000 \mathrm{~cm}^{-1}$. On atteint la limite de résolution de l'appareil.

Pendant l'arrêt du monochromateur, les mesures pourront être effectuées de deux façons:

- soit, sans déplacement du faisceau, grâce au secteur à cristaux ou à la translation du cryostat (cas des mesures à basse température) :

- soit, avec déplacement du faisceau, par l'intermédiaire du disque tournant seul, ou de l'ensemble disque tournant-secteur à cristaux.

b) La synchronisation des mouvements des dispositifs de mesures avec l'entraînement pas à pas.

Par cet ensemble très complet, il est ainsi possible d'effectuer tous types de mesures.

\section{PERFORMANCES DE L'APPAREIL}

Les qualités d'un monochromateur sont essentiellement jugées sur sa résolvance et sa luminosité obtenues suivant les domaines spectraux utilisables, sur l'absence de lumière parasite, sur sa précision, la reproductibilité du système de défilement des longueurs d'onde, ainsi que sur la qualité des dispositifs de mesures.

I. Résolvance et luminosité. - Il est bien connu que pour une résolvance donnée la luminosité du monochromateur utilisant le montage LITTROW est maximum lorsque les fentes d'entrée et de sortie ont des largeurs identiques.

C'est ce qui est réalisé dans notre cas.

La résolvance pratique:

$$
R_{\mathrm{u}} \leqslant \frac{F}{e} \lambda \frac{\mathrm{d} \theta}{\mathrm{d} \lambda}
$$

prend ici l'expression:

$$
R_{\mathrm{u}} \leqslant 2 \frac{F}{e} \operatorname{tg} \theta
$$

à largeur de fente $e$ constante, il augmente donc avec la distance focale du miroir collimateur (ici $F=750 \mathrm{~mm}$ ) et l'angle de diffraction $\theta(\lambda)$. Ce qui montre l'intérêt d'utiliser des réseaux à angle de miroitement $\varphi$ important $\left(\varphi \approx 27^{\circ}\right.$ et $\theta \approx \varphi \pm 10^{\circ}$ ).

Le tableau suivant précise les conditions d'emploi de l'appareil (réseau, prisme, ordre utilisé) et donne les valeurs, calculées et pratiquement observées pour les trois réseaux, du facteur $R_{\mathrm{u}} \cdot e_{\mathrm{mm}}$ (résolvance multipliée par la largeur de fente exprimée en $\mathrm{mm}$ ), dans l'intervalle $1 \mu$-33 $\mu$. 


\section{Réseau I}

292 traits $/ \mathrm{mm}$

miroitement $26^{\circ} 1^{\prime}$

Prisme : $\mathrm{NaCl}$

\section{Réseau II}

75 traits $/ \mathrm{mm}$

miroitement $26^{\circ} 45^{\prime}$

Prisme : $\mathrm{NaCl}$

\section{Réseau III}

40 traits $/ \mathrm{mm}$ miroitement $26^{\circ} 45^{\prime}$ $15-20 \mu$ : Prisme $\mathrm{KBr}$ 20-30 $\mu$ : Prisme CsBr

$\begin{array}{rrrrrrrr}\lambda_{\mu} & 1 & 1,5 & 2 & 3 & 4 & 4,5 & \\ & & & 450 & 730 & 1080 & 1300 & 1^{\text {er }} \text { ordre } \\ R_{\mathrm{u}} \cdot e_{\mathrm{mm}} & 730 & 730 & 1080 & & & & 2^{\mathrm{e}} \text { ordre }\end{array}$

$\begin{array}{lllllll}\lambda_{\mu} & 5 & 6 & 8 & 10 & 12 & 15\end{array}$

$\begin{array}{rrrrrrrr} & & 460 & 610 & 750 & 1020 & 1^{\text {er ordre }} \\ R_{\mathrm{u}} \cdot e_{\mathrm{mm}} & 610 & 750 & 1130 & & & & 2^{\mathrm{e}} \text { ordre }\end{array}$

$\begin{array}{rrrrrr}\lambda_{\mu} & 15 & 18 & 20 & 25 & 30 \\ R_{\mathrm{u}} \cdot e_{\mathrm{mm}} & 465 & 580 & 660 & 860 & 1020\end{array}$

$1^{\mathrm{er}}$ ordre
De ce tableau, on peut tirer les valeurs de l'intervalle spectrai que l'on sépare avec des fentes de largeur donnée (variable de 0 à $4 \mathrm{~mm}$ ).

Dans des conditions normales (rapport signal/ bruit voisin de 460 ), on obtient une résolution

- avec le réseau I :

$$
\text { à } 3300 \mathrm{~cm}^{-1} \text { de } \Delta v=0,4 \mathrm{~cm}^{-1} \text {, }
$$

- avec le réseau II :

$$
\text { à } 830 \mathrm{~cm}^{-1} \text { de } \Delta v=0,25 \mathrm{~cm}^{-1},
$$

- avec le réseau III :

$$
\text { à } 500 \mathrm{~cm}^{-1} \text { de } \Delta v=0,3 \mathrm{~cm}^{-1} \text {. }
$$

La figure 5 représente, avec un rapport signal/bruit voisin de 60, l'enregistrement de la région 1045 $1095 \mathrm{~cm}^{-1}$ de la bande fondamentale de l'ammoniac (largeur spectrale de fente : $0,15 \mathrm{~cm}^{-1}$ ). Nous donnons aussi la région centrale de la bande à $4,257 \mu$ du gaz carbonique atmosphérique (Fig. 6).

Tous les spectres ont été obtenus avec des récepteurs pneumatiques type GOLAY UNICAM à fenêtre KRS 5 et diamant (diamètre $3 \mathrm{~mm}$ ) ayant une détectivité de l'ordre de $2 \times 10^{5}$ volts/watts, en liaison avec l'unité amplificatrice SP 50 et un enregistreur SEFRAM GRAPHISPOT type GRVAT.

II. Pureté des spectres. Précision et reproductibilité des longueurs d'onde. - La proportion de lumière parasite est très faible ; elle peut résulter d'un chevauchement partiel des ordres, du fait d'un élargissement important des fentes. Ceci se traduit, par exemple à $800 \mathrm{~cm}^{-1}$ par l'existence autour du pic correspondant au 1 er ordre, d'un taux inférieur à $0,5 \%$ de lumière de longueur d'onde différente.

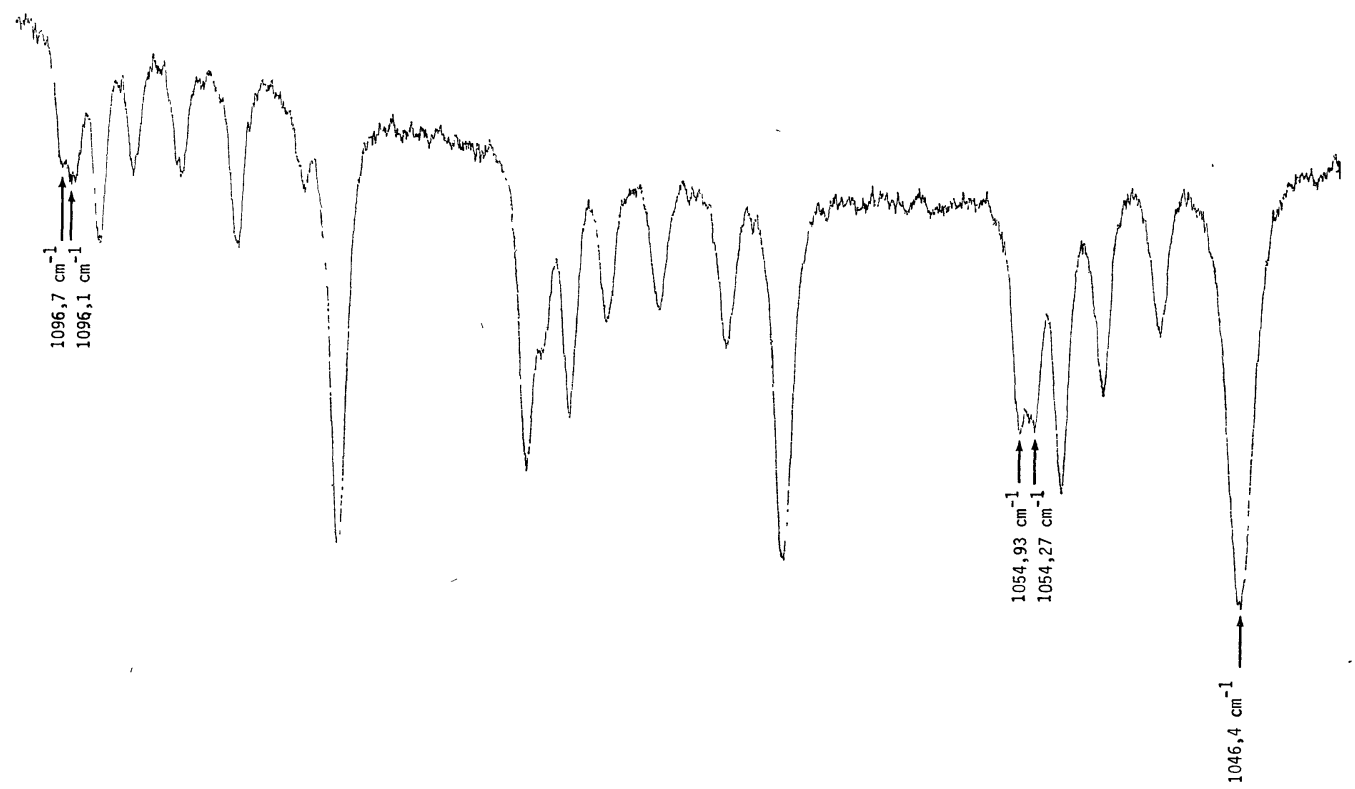

Fig. 5. - Région $1045-1095 \mathrm{~cm}^{-1}$, bande fondamentale $v_{2}$ de l'ammoniac. Cellule $10 \mathrm{~cm}$ avec fenêtres $\mathrm{NaCl}$. Réseau 75 traits $/ \mathrm{mm}, 1^{\text {er }}$ ordre. Vitesse de défilement $1 \mathrm{~cm} / \mathrm{mn}$. Largeur spectrale de fente : $0,15 \mathrm{~cm}^{-1}$. 


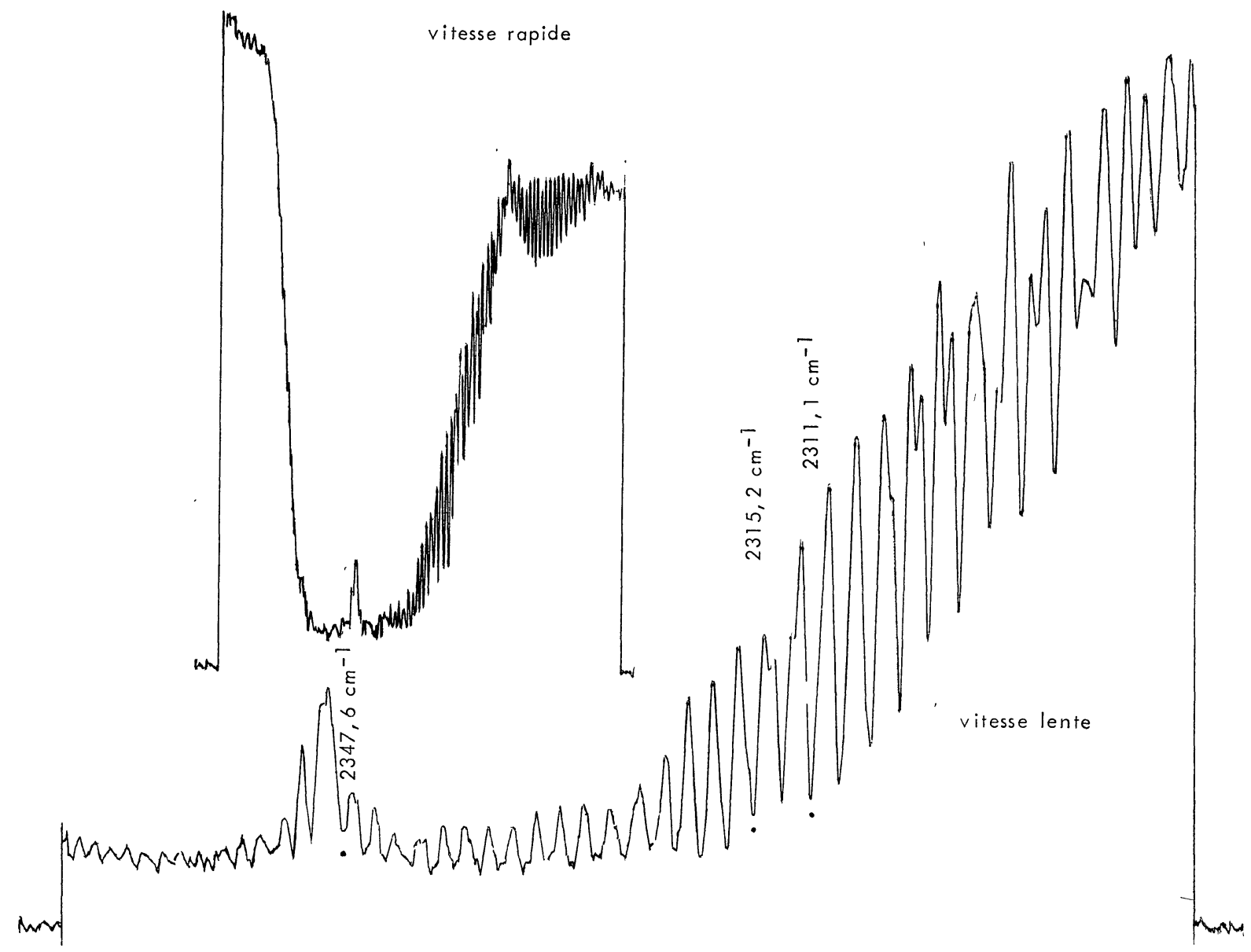

FIG. 6. - Région centrale de la bande à $4,257 \mu$ du gaz carbonique atmosphérique. Réseau 300 traits/mm, largeur spectrale des fentes $0,5 \mathrm{~cm}^{-1}$. Vitesse de défilement $6 \mathrm{~cm} / \mathrm{mn}$.

Ce taux qui s'est révélé légèrement plus important seulement dans la zone $380-480 \mathrm{~cm}^{-1}$, a été totalement annulé $(\ll 0,1 \%)$ par le remplacement du miroir $\mathrm{M}_{10}$ par une lame de fluorure de lithium réfléchissant $90 \%$ du flux dans cette région. La reproductibilité en longueur d'onde d'un spectre, quelle que soit la longueur de l'enregistrement, est très bonne (meilleure que $0,001 \mu$ à $3 \mu$ ). Le changement de réseau ou de prisme n'entraîne pas de variation de l'étalonnage.

Le système de linéarisation en longueur d'onde, réglé optiquement avec une précision de l'ordre de $30^{\prime}$ d'angle, implique la possibilité de variation de linéarité de l'ordre de $\frac{\Delta \lambda}{\lambda}=\frac{3}{1000}$.

L'étalonnage du spectromètre, effectué avec les bandes de vibration-rotation de $\mathrm{CO}_{2}, \mathrm{H}_{2} \mathrm{O}, \mathrm{NH}_{3}$, permet de déterminer des variations de linéarité, sur l'intervalle de longueur d'onde correspondant à un ordre, voisines de $\frac{\Delta \lambda}{\lambda}=\frac{2}{1000}$. Ceci n'est pas, gênant dans le cas des mesures courantes. Dans le cas de mesures très précises, une interpolation entre les raies d'un spectre connu est nécessaire, la linéarité sur un intervalle court permettant d'atteindre alors une précision de détermination même supérieure à la résolution.

Quant au défilement discontinu du spectromètre, les intervalles spectraux choisis sont conservés sur un spectre durant plusieurs heures.

III. Principaux travaux originaux déjà effectués. Les principaux travaux originaux déjà effectués à l'aide de cet appareil portent sur l'étude de l'anharmonicité des cristaux ioniques et moléculaires, sur des mesures de biréfringence, sur les structures cristallines en liaison étroite avec les spectres Raman et la diffraction des rayons $\mathrm{X}$.

Dans le premier domaine, nous citerons les travaux portant sur le comportement des spectres d'absorption multiphonon du $\mathrm{MgF}_{2}$ (Fig. 7), [9], [10] et des spectres de réflexion (incidence $6^{\circ}$ ) du $\mathrm{LiCH}_{3} \mathrm{COO}$, $2 \mathrm{H}_{2} \mathrm{O}$ (Fig. 8), [11], [12], lorsque la température varie de $6^{\circ} \mathrm{K}$ à $300^{\circ} \mathrm{K}$.

La qualité et les facilités d'utilisation du spectromètre ont permis d'atteindre outre les valeurs très précises à $0,8 \%$ près des biréfringences principales $d u$ soufre $\alpha$ entre 3 et $13 \mu$, des résultats importants relatifs à l'effet d'une pression uniaxiale sur celles-ci (valeur élevée des coefficients piézo-optiques, modulation 


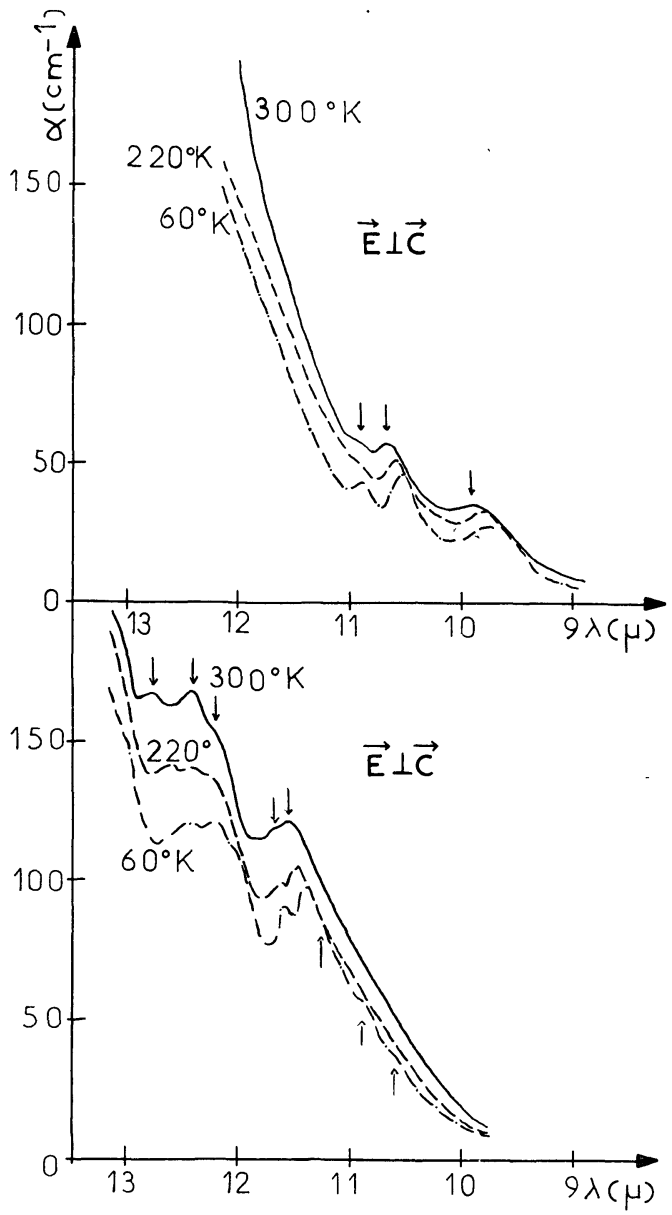

FIG. 7. - Coefficient d'absorption de $\mathrm{MgF}_{2}$ : mesuré pour des polarisations parallèle et perpendiculaire à l'axe optique de $9^{\circ} \mathrm{K}$ à $300^{\circ} \mathrm{K}$. Réseau $75 \mathrm{t} / \mathrm{mm}$, largeur spectrale $5 \mathrm{~cm}^{-1}$; lame de $0,29 \mathrm{~mm}$ d'épaisseur.

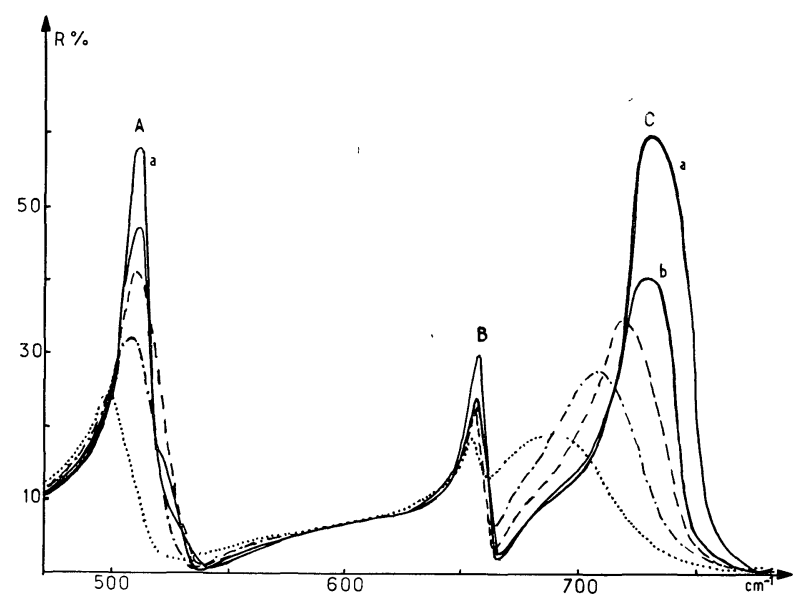

FIG. 8. - Monocristal d'acétate de lithium. Effets de température sur la dispersion infrarouge, zone de $450-750 \mathrm{~cm}^{-1}$, direction $\mathrm{E}$ à $y$, angle d'incidence $6^{\circ}$, largeur spectrale $2 \mathrm{~cm}^{-2}$, vitesse de défilement de 5 à $10 \mathrm{~cm}^{-1}$ par $\mathrm{mn}$.
A : Mode de valence antisymétrique. Li-O ;
B : Mode déformation symétrique $\mathrm{COO}\left(v_{5}\right)$;
$\mathrm{C}$ : Móde de rotation plane $\mathrm{H}_{2} \mathrm{O}(2)$;

a) $\left.: 6^{\circ} \mathrm{K}-26^{\circ} \mathrm{K}, b\right): 83^{\circ} \mathrm{K}, \ldots: 140{ }^{\circ} \mathrm{K}, \cdots, .: 220^{\circ} \mathrm{K}, \ldots: 295^{\circ} \mathrm{K}$
$100 \%$ de l'intensité d'un faisceau monochromatique) (Fig. 9), [8], [13].

Enfin les spectres inédits de réflexion en lumière polarisée ont été obtenus sur de nombreux cristaux,

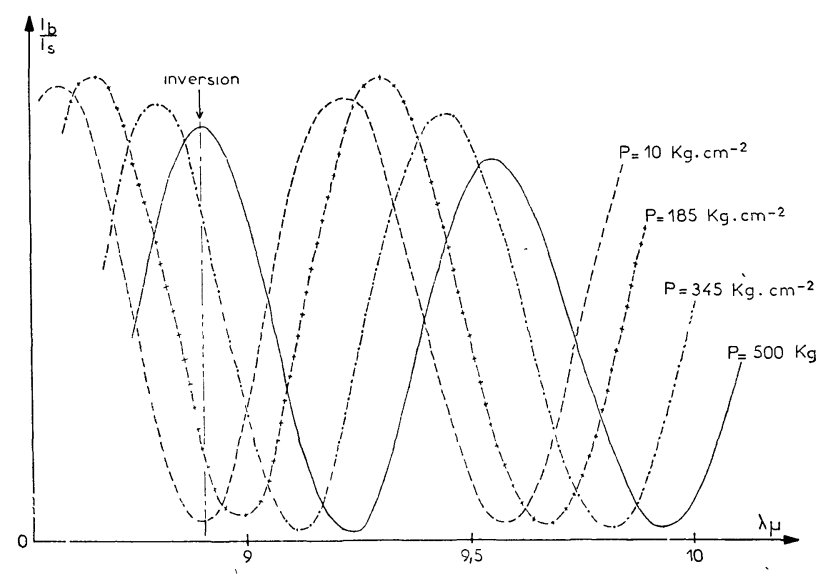

FIG. 9. - Déplacement des franges de biréfringence du soufre $\alpha$ sous l'effet d'une pression uniaxiale parallèle à b (lame 001, épaisseur $2,485 \mathrm{~mm}$, largeur spectrale de fente $1,5 \mathrm{~cm}^{-1}$, $\left.T=293^{\circ} \mathrm{K}\right)$.

en particulier des carboxylates de lithium [14] ou d'ammonium [15], des hypophosphites [16] et des sulfates de divers métaux. La précision de détermination des maximums de pouvoir réflecteur est de l'ordre de $3 \%$.

Dans certains cas, ces mesures ont permis de rejeter les structures précédemment établies et d'émettre de nouvelles hypothèses, en particulier [11], vérifiées par la suite.

Conclusion. - Les performances du monochromateur se sont révélées conformes aux critères, que nous nous étions imposés. Comparables à celles des meilleurs appareils à fentes actuels, elles sont dues pour une part importante au montage optique, plus précisément à la position des miroirs centraux $\mathbf{M}_{4}$ et $\mathbf{M}_{6}$, permettant une mise au point précise de l'ensemble et un minimum d'aberrations.

Les solutions adoptées pour interchanger rapidement les différents organes (réseaux, prismes) ou programmer le type d'exploration du spectre (continu, point par point) lui confèrent une grande souplesse d'emploi et une reproductibilité parfaite des résultats obtenus dans le domaine $300-10000 \mathrm{~cm}^{-1}$.

A tout ceci s'ajoute une versatilité totale, quant à l'adaptation rapide de tout nouveau module de mesures. Sa conception par blocs a rendu particulièrement simple l'adjonction de nombreux dispositifs (disque à cristaux, cryostat à température variable, presse, ...) permettant des mesures précises en réflexion, transmission ou émission dans différentes conditions physiques. 


\section{Bibliographie}

[1] Deloupy (Cl.), Vergnoux (A. M.), Rouguayrol (F.), Revue Opt., 1957, 36, 20 ; 1958, 37, 113.

[2] Duverney (R.), Thèse, Montpellier, 1963.

[3] Ford (M. A.), Price (W.), Wilkinson (G. R.), Journal of Sci. Instr., 1958, 35, 55.

[4] Vedel (C.), BouHet (Ch.), Revue Opt., 1963, 7, 361.

[5] Hadni (A.), J. Physique Rad., 1962, 23, 152. Ann. Phys., 1956, 13 e série, 1, 234.

[6] JACQUinOT (P.), Revue Opt. Théor. Instrum., 1954, 33, 653.

[7] BousQuet (P.), Spectroscopie Instrumentale, Paris, Dunod, 1969.
[8] Almairac (R.), Thèse $3^{\mathrm{e}}$ cycle, Montpellier, 1971.

[9] Benoit (Cl.), Thèse, Montpellier, 1969.

[10] Benoit (Cl.), J. Physique, 1970, 31, 1005.

[11] CaDene (M.), Spectroch. Acta, à paraître.

[12] Cadene (M.), Thèse, Montpellier, 1969.

[13] Almairac (R.) et Vergnoux (A. M.), Comptes rendus, 1971, 272, 1442.

[14] Cadene (M.), Comptes rendus, 1969, 268, 1079.

[15] Fournel (A.) et Vergnoux (A. M.), Comptes rendus, 1971, 272, 1381.

[16] Abenoza (M.), Hillaire (P.) et Cadene (M.), Comptes rendus, 1970, 271, 346. 